\title{
Effect of Parameters of Isopipe on the Quality of Glass Sheet Produced from Overflow Fusion Process by Numerical Simulation
}

\author{
Yansheng Hou ${ }^{1}$, Jinshu Cheng ${ }^{1,2}$, Junfeng Kang ${ }^{1}$, Jingjing Cui ${ }^{1}$, Xinjian Xue ${ }^{3}$ and Dong Zuo ${ }^{3}$ \\ ${ }^{1}$ State Key Laboratory of Silicate Materials for Architectures, Wuhan University of Technology, Wuhan, 430070, China \\ ${ }^{2}$ Hebei Shahe Glass Technology Research Institute, Shahe, 054100, China \\ ${ }^{3}$ Shaanxi Caihong Electronic Glass Company Limited, Xianyang, 712000, China
}

\begin{abstract}
A numerical model for simulation of molten glass flow with semi-implicit method for pressure-linked equations (SIMPLE) and Volume of fluid (VOF) method during the overflow fusion process was carried out by using FLUENT software. The effect of the geometry parameters of the isopipe and flow rate of molten glass on the flow patterns during overflow was investigated. The results showed that the overflow trough depth only had an effect on the flow rate distribution of glass melt around the inlet point. The tilt angle at the top of the trough had a significant effect on flow rate distribution along the length of the trough, and the desired mass distribution was achieved at the tilt angle $4^{\circ} \sim 6^{\circ}$. The flow rate had a large influence on the overflow of the molten glass, which had a directive significance to the overflow down draw sheet manufacturing process.
\end{abstract}

\section{Introduction}

Currently, the glass used as LCD substrates are produced by two different processes: floating process [1], and overflow fusion process [2]-[5]. One of the major advantages of the overflow fusion process is that the surfaces of the resulting glass sheet are pristine without any contact with refractory material during forming. As a result, the overflow fusion process has become a particular importance method in the production of the glass substrates used in the manufacture of LCDs.

To gain a better understanding of molten glass flow during the overflow fusion process, the physical phenomena should be well studied. Therefore, in this study, FLUENT software was used to simulate the 3D flow of the molten glass in the isopipe. A finite different volume of fluid (VOF) approach for tracking the waving of the surface of the molten glass was proposed. The effect of the geometry of the isopipe on the flow patterns during overflow was investigated to obtain the relationships among flow pattern and geometry of the isopipe. Then, an optimized design of isopipe could be achieved. In addition, flow rate of molten glass on the flow patterns during overflow were also studied, which had a certain guiding role for actual production.

\section{Physical model}

The molten glass was conducted through an inlet pipe to the sheet forming apparatus, which was formed by a refractory material known as an "isopipe". The schematic diagram of the isopipe is shown in Fig. 1. After filling the trough of the isopipe, the molten glass flowed over both side walls of the trough (called weirs), down along the side surfaces of the isopipe as two glass ribbons. The two sheets met at the root of the isopipe, where they fused together into a single sheet. The single sheet was then fed to a drawing equipment, which controlled the thickness of the sheet by the rate at which the sheet was drawn away from the root.
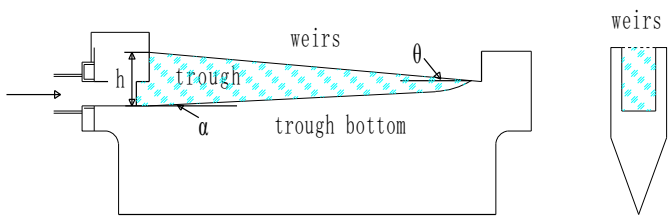

Figure 1. The schematic diagram of the isopipe for the overflow process.

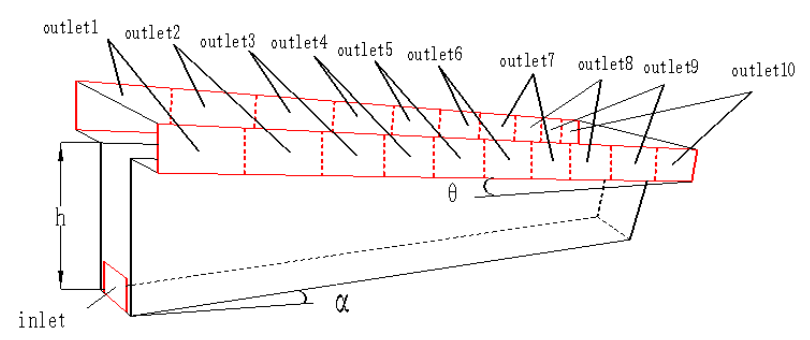

Figure 2. Simulation model of the trough.

The process of molten glass flowing in the isopipe included three parts. The molten glass was conducted through the inlet pipe to the isopipe ( $\mathrm{Y}$ direction), and then the molten glass rose in vertical direction ( $\mathrm{Z}$ direction). At last the molten glass overflowed from the weir surfaces $(\mathrm{X})$. Due to the stresses from $\mathrm{X}, \mathrm{Y}$, and Z 
three directions, so the geometry was meshed using 3D GAMBIT software with hexahedral cells. In order to make the analysis easier, each side of overflow weirs was average divided into 10 outlets (shown as Fig. 2). Therefore, the glass melt flow distribution at top of the trough along the length of the isopipe could be calculated respectively.

\section{Simulation details}

In this paper, the powerful FLUENT software was used to simulate the overflow fusion process. SIMPLE algorithm was selected in the calculation process. The surface tracking of the molten glass-air interface was studied by VOF model with open channel method [6].

In order to simulate the molten glass flowing in the trough, some assumptions were proposed [7]:

1 . The molten glass with high viscosity had low Reynolds numbers, assuming that the fluid flow was a laminar flow motion.

2. The molten glass was stable without component unevenness, chemical reaction, bubbles, stones and so on.

3. The molten glass was a uniform incompressible Newtonian fluid.

4. The temperature of the molten glass was considered as a constant without heat transfer, that is, the thermal conductivity $C_{p}$ was 0 .

Some physical properties of the molten glass were approximately constant, including density, viscosity, coefficient of expansion.

\subsection{Governing equations}

The molten glass was approximatively considered as an incompressible viscous fluid. Therefore the density was equal to a constant in the incompressible Navier-Stokes equation. The continuity equation [8]:

$$
\nabla \mathbf{V}=0
$$

Suppose the viscosity was equal to a constant in the entire flow process. The momentum equations:

$\mathrm{X}$ direction momentum equation:

$$
\rho \frac{\mathrm{D} u}{\mathrm{D} t}=-\frac{\partial p}{\partial x}+\eta \nabla^{2} u+\rho f_{x}
$$

$\mathrm{Y}$ direction momentum equation :

$$
\rho \frac{D v}{D t}=-\frac{\partial p}{\partial y}+\eta \nabla^{2} v+\rho f_{y}
$$

$\mathrm{Z}$ direction momentum equation:

$$
\rho \frac{\mathrm{D} w}{\mathrm{D} t}=-\frac{\partial p}{\partial z}+\eta \nabla^{2} w+\rho f_{z}
$$

Assuming the temperature $T$ was a constant, the viscosity $\eta$ was derived as a constant (because it is a function of $T$ ). The four equations(1) (4) are closed, which have four equations and four unknowns $u, v, w$, and $p$. Assuming the $\rho=$ constant,$\eta=$ constant, the energy equation is decoupled. Therefore the continuity equation and momentum equation were used to solve the problems of molten glass flowing process.

\subsection{Treatments for the free surface flow}

VOF model can be used to model two or more immiscible fluids. This is done by solving a single set of momentum equations and by tracking the volume fraction of both the liquids-air throughout the domain.

The volume fraction equation [9]:

$\frac{1}{\rho}\left[\frac{\partial}{\partial t}\left(\alpha_{q} \rho_{q}\right)+\nabla\left(\alpha_{q} \rho_{q} \mathbf{v}_{\mathbf{p}}\right)=S_{\alpha_{q}}+\sum_{p=1}^{\mathrm{n}}\left(m_{p q}-m_{q p}\right)\right]$

Here $\rho_{q}$ is the density, $\mathbf{v}_{\mathbf{p}}$ is the velocity and $\alpha_{q}$ is the volume fraction of the $q$ phase. $m_{p q}$ and $m_{q p}$ are mass flow rates of phases $p$ to $q$ and $q$ to $p$ respectively. The volume fraction of secondary phases is computed from the equation below:

$$
\sum_{q=1}^{n} \alpha_{q}=1
$$

\subsection{Parameters of the model materials}

The various material properties are defined (as shown in Table 1) and then suitable boundary (as shown in Table 2) conditions are applied. The parameters of glass melt in simulation process were determined by practical production process. The three most important geometric parameters of the isopipe were the depth $(h)$ of the inlet point, the bottom tilt angle $(\alpha)$ of the trough and the tilt angle $(\theta)$ of the overflow weirs. Different flow rates of molten glass on the flow patterns during overflow were also investigated. The detail parameters used in this study are shown in Table 3.

Table 1. Materials properties.

\begin{tabular}{|c|c|c|}
\hline Material & Physical property & Value \\
\hline \multirow{3}{*}{ Molten glass } & Density & $2500 \mathrm{~kg} / \mathrm{m}^{3}$ \\
\cline { 2 - 3 } & Viscosity & $1750 \mathrm{~Pa} \cdot \mathrm{s}$ \\
\cline { 2 - 3 } & Surface tension & $300 \mathrm{dyne} / \mathrm{cm}$ \\
\hline \multirow{2}{*}{ Air } & Density & $1.225 \mathrm{~kg} / \mathrm{m}^{3}$ \\
\cline { 2 - 3 } & Viscosity & $1.784 \times 10^{-5} \mathrm{~Pa} \cdot \mathrm{s}$ \\
\hline
\end{tabular}

Table 2. Boundary conditions.

\begin{tabular}{|c|c|}
\hline Contents & Specification \\
\hline \multirow{2}{*}{ Inlet } & Velocity Inlet \\
\cline { 2 - 2 } & Molten glass volume fraction $=1$ \\
\hline \multirow{3}{*}{ Outlet } & Pressure Outlet \\
\cline { 2 - 2 } & Gauge pressure $=0$ Pa \\
\cline { 2 - 2 } & Backflow volume fraction $=0$ \\
\cline { 2 - 2 } & Open channel flow specification \\
\hline
\end{tabular}

Table 3. Parameters for simulation.

\begin{tabular}{|c|c|c|c|c|}
\hline & $\begin{array}{c}\text { Height of } \\
\text { trough at inlet } \\
\text { end }(\mathrm{cm})\end{array}$ & $\begin{array}{c}\text { Tilt angle } \\
\text { of trough } \\
\text { bottom } \alpha\left(^{\circ}\right)\end{array}$ & $\begin{array}{c}\text { Tilt angle of } \\
\text { weir surface } \\
\theta\left(^{\circ}\right)\end{array}$ & $\begin{array}{c}\text { Flow } \\
\text { velocity } \\
(\mathrm{kg} / \mathrm{h})\end{array}$ \\
\hline Model 1 & $\begin{array}{c}15,20,25,30, \\
35,40,50\end{array}$ & 0 & 0 & 300 \\
\hline Model 2 & 40 & $1,2,3,4,5,6$ & 0 & 300 \\
\hline Model 3 & 40 & 0 & $1,2,3,4,5,6,7$ & 300 \\
\hline
\end{tabular}




\section{Simulation results and discussion}

\subsection{The effect of geometric parameters of overflow trough}

Due to the importance of stability in production process, the numerical studies were carried out under steady conditions, and then obtained the steady state of fluid at different initial conditions. Each model was calculated iteratively under the initial conditions until the residual standard error remained unchanged (the difference in mass between inlet and outlet was less than $0.01 \%$ ). The flow of the overflow glass melts was obtained by calculating that of the ten outlet (evenly distributed), then the mass distribution of three models were obtained, as shown in Figs. 3, 4 and 5.

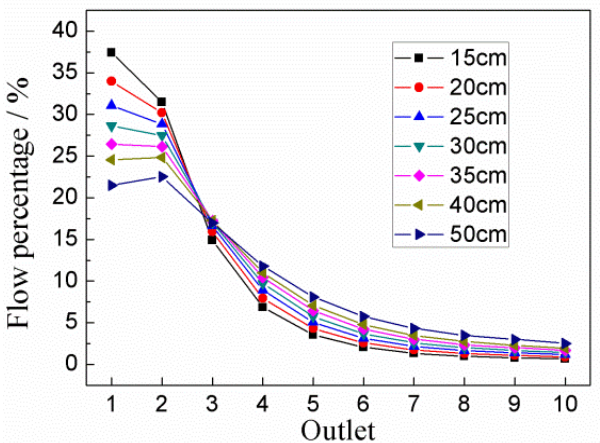

Figure 3. Mass distribution of glass melt with different height values of trough.

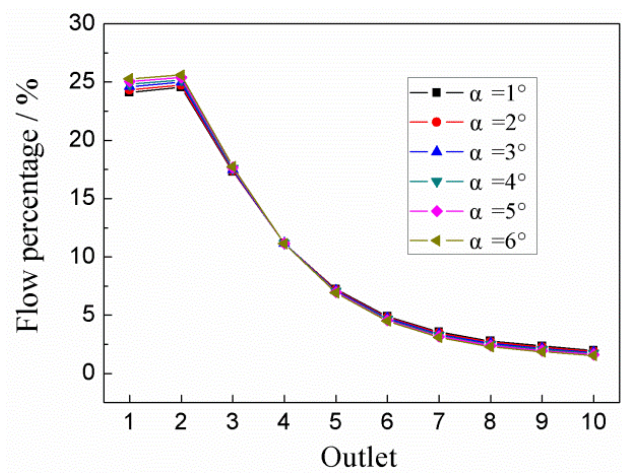

Figure 4. Mass distribution of glass melt with different tilt angle of trough bottom.

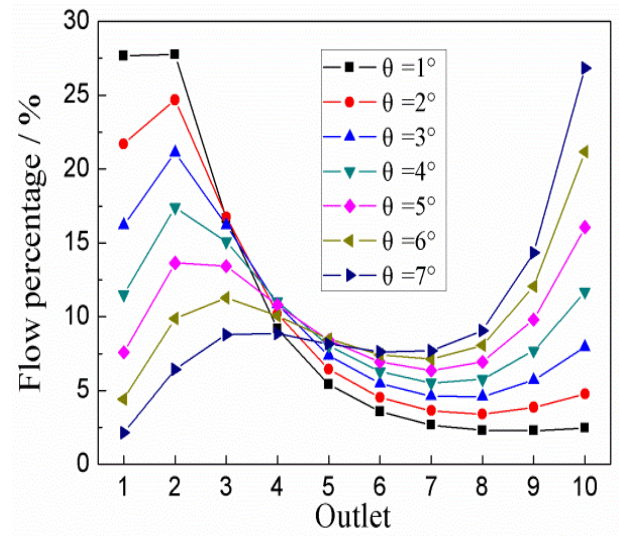

Figure 5. Mass distribution of glass melt with different tilt angle of trough surface.
The flow of the overflow molten glass with different values of $h$ and $\alpha$ was shown in Fig. 3 and Fig. 4. The results showed that the flow of molten glass at inlet point was greater than that of the far end. As shown in Fig. 3, the depth of overflow trough $(h)$ influenced molten glass flow at inlet point greatly. The molten glass flow (within $1 / 5$ of the length of the inlet point) decreased rapidly when $\mathrm{h}$ increased, but $\mathrm{h}$ had negligible impact to the rest part. The flow distribution curves of molten glasses with different values of $\alpha$ were almost overlapped (as shown in Fig. 4), which indicated that the change of bottom tilt angle of trough did not make a significant difference in the flow distribution of molten glass.

As shown in Fig. 5, with the overflow surface tilt angle $(\theta)$ gradually increased, the flow distribution of molten glass in the overflow surface changed significantly. With the value of $\theta$ increasing, the flow of molten glass at the inlet point reduced, and reduction of the level height of the overflow surface along the extent of the trough provided the possibility of low potential outflow at the far end. With the tilt angle $\theta$ increasing from $4^{\circ}$ to $6^{\circ}$, the flow rate distribution curve close to a smooth level, particularly between outlet 2 and outlet 8 . Although the molten glass mass distribution curve is still not very even, the distribution that the large overflow in the both ends and small overflow in the middle can meet the actual production needs. Because when the glass overflow down, the non-flowing width of the edge of the glass requires a stretching rolls. In order to ensure the strength of the edges, the thickness of the edges should be increased, so it is necessary to increase the flow in the both edges.

\subsection{The effect of flow rate}

According to the above results, the optimal geometry model is that: the bottom tilt angle is 0 , overflow surface tilt angle is $5^{\circ}$, the depth of trough at the inlet point is $0.4 \mathrm{~m}$, and the length of trough is $2 \mathrm{~m}$. The effect of velocity on the mass distribution of the molten glass was investigated, shown in Fig. 6.

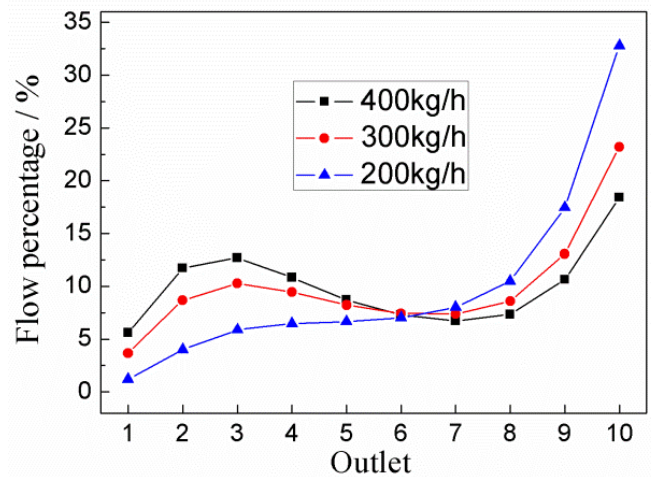

Figure 6. Mass distribution of glass melt with different flow rate.

In model 4, the initial speed of glass melt has greatly influenced on the mass distribution overflow from the weir surfaces, as showed in Fig. 6. The most even flow rate distribution in middle area of trough was obtained with the initial speed of $300 \mathrm{~kg} / \mathrm{h}$. In addition, since the 
mass distribution of glass melt was dependent on the flow rate, during practical production, when optimum process had been determined, the viscosity and speed could not be changed casually. If different thickness of glass is wanted (e.g. $0.3 \mathrm{~mm}, 0.4 \mathrm{~mm}$, and $0.5 \mathrm{~mm}$ ), the only factor that can be adjusted is the pulling speed, not the flow rate of glass melt, for fast speed could get thinner glass. This method of control the thickness of glass is a basic adjustment way during overflow down-draw process of glass industry, under the condition of a steady flow rate.

\section{Summary}

After the above modeling, calculation and analysis, some conclusions were obtained. The tilt angle of weir surface $\theta$ was the most important factor of the geometry parameters of the isopipe, which had a significant effect on flow rate distribution of the glass melt along the length of the trough, and the desired mass distribution was achieved at the tilt angle $4^{\circ} \sim 6^{\circ}$. Height of trough at inlet end and tilt angle of trough bottom had little effect on the glass overflow mass distribution, but the groove depth only had some influence on mass distribution around the inlet point. With the increase of inlet velocity, the flow rate of the glass melt decreased at the inlet point, and increased at the distal end.

\section{Acknowledgement}

This work was supported by the National Key Research and Development Program of China (2016YFB0303702).

\section{References}

1. C.Y. Wang, Q. Lu, Glass \& Enamel 3, 44 (2016).

2. R.P. William, P.R. Kamjula, Chinese Patent Number CN 101925545 A, September 12, (2010).

3. S.M. Milillo, R.L. Rhoads, United States Patent Number US8973402, October 24, (2011).

4. K.W. Aniolek, S.R. Burdette, L.R. de Paor, et al. United States Patent Number US8393178, October 18, 2011.

5. R. Delia, European Patent Number EP 2253598 B1, May 21, 2010.

6. C.W. Hirt, B.D. Nichols, J. Comput. Phys. 39, 1 (1981).

7. H.J. Lin, F.Y. Hsu, W.K. Chang, Adv. Mater. Res. 39-40 (2008).

8. J. Abbott, 26th Annual Workshop on Mathematical Problems in Industry Rensselear Polytechnic Institute (2010).

9. A. Tiwari, S.S. Mondal, P. Kumar, et al, Adv. Mech. Eng. 1, 516 (2011). 